assessment of cSLE activity (MD-global), disease activity index score, urine protein to creatinine ratio (PCR), Child Health Questionnaire physical function summary score (CHQ-Phs)]. Percentage changes of these cSLE-CRVs are used in the Provisional PRINTO-ACR- EULAR Criteria of Response to Therapy of cSLE (PCI). In a small dataset, we have previously shown that the PCI and the Systemic Lupus Responder Index only have fair accuracy in detecting cSLE improvement. The Objective of this research was to 1) validate the PCI and 2) develop for Children an Index of Lupus Improvement (CHILI) as a tool to measure response to therapy, with focus on clinically relevant improvement (CRI $\mathrm{CSLE})$.

Methods Pediatric subspecialists $(n=213)$ in treating cSLE were invited to define $\mathrm{CRI}_{\mathrm{CSLE}}$ and rate a total of 433 unique patient profiles for the presence/absence of $\mathrm{CRI}_{\mathrm{CSLE}}$ and various levels of improvement. Patient profiles included the cSLECRVs and routine laboratory tests at a baseline and follow-up time-point. To measure $\mathrm{CRI}_{\mathrm{CSLE}}$ we tested the PCI, and developed the candidate CHILI criteria that considered a) absolute and b) percentage changes of the cSLE-CRVs (baseline vs follow-up) in a trainings-dataset and initially validated these criteria in the validation-dataset. Criteria accuracy was assessed by kappa statistics (PCI) and the area under the ROC curve (AUC; range: 0-1)], respectively.

Results During an international consensus conference agreement on a definition of $\mathrm{CRI}_{\mathrm{cSLE}}$ was achieved. Response rate to patient profile ratings was 91\% (194/214). The PIC had no more than fair accuracy (kappa <0.43). There was consensus (92.3\%) that the CHILI is preferable to the PCI to measure $\mathrm{CRI}_{\mathrm{CSLE}}$. As shown in the figure 1, considering absolute changes of the cSLE-CRVs, a CHILI score of $\geq 54$ (range 0100) was highly accurate for identifying $\mathrm{CRI}_{\mathrm{CSLE}}$ (AUC=0.93; sensitivity $=81.1 \%$; specificity $=84.2 \%$ ) in the validation-dataset. Likewise CHILI scores exceeding 15, 68 and 92 reflected minor, moderate and major improvement for values (all: AUC >0.92, sensitivity: >93.1\%; specificity: >73.4\%), respectively.

Conclusions The CHILI is a new highly accurate index to capture improvement in the overall course of cSLE. This index is also useful to categorize the degree of cSLE response to therapy. Acknowledgements For the Pediatric Rheumatology International Trial Network and the Pediatric Rheumatology Collaborative Study Group; the study is supported by NIH grants 5U01-AR51868, P30-AR AR47363 and 2UL1RR026314 and the PRCSG and PRINTO Coordinating Centers. This study is also supported by grants from Fundação de Amparo à Pesquisa do Estado de São Paulo (FAPESP 2015/03756-4 to CAS), Conselho Nacional de Desenvolvimento Científico e Tecnológico (CNPq 303422/2015-7 to CAS) and by Núcleo de Apoio à Pesquisa 'Saúde da Criança e do Adolescente' da USP (NAP-CriAd) to CAS.

\section{CS-11 THE EFFECTS OF CONTEXTUAL SOCIODEMOGRAPHIC FACTORS AND HEALTH RESOURCE CONCENTRATION ON HYDROXYCHLOROQUINE ADHERENCE AMONG U.S. MEDICAID BENEFICIARIES WITH LUPUS}

${ }^{1}$ Candace H Feldman* ${ }^{1}$ Karen H Costenbader, ${ }^{1}$ Daniel H Solomon, ${ }^{2}$ SV Subramanian, ${ }^{2}$ Ichiro Kawachi. 'Division of Rheumatology, Immunology and Allergy, Department of Medicine, Brigham and Women's Hospital, Boston, MA, USA; ${ }^{2}$ Department of Social and Behavioral Sciences, Harvard T.H. Chan School of Public Health, Boston, MA, USA

10.1136/lupus-2018-Ism.46
Background Adherence to hydroxychloroquine (HCQ), the standard-of-care treatment among patients with systemic lupus erythematous (SLE) is suboptimal and interventions to date to improve this have been largely unsuccessful. Individual-level factors, including younger age and non-white race/ethnicity, have been implicated. While contextual influences such as area-level poverty are known to contribute to health behaviors and to SLE-related damage, no studies to date investigate the role of these factors on HCQ adherence. We therefore aimed to use multilevel models to understand the effects of sociodemographic variables (area-level racial composition, poverty, educational attainment) and health resource concentration (numbers of physicians, pharmacists, hospitals) on HCQ adherence while also accounting for potential individual-level factors.

\begin{tabular}{|c|c|c|}
\hline Area-level sociodemographics and health resources & $\begin{array}{l}\text { Odds ratio } \\
\text { (OR) }\end{array}$ & $\begin{array}{l}95 \% \text { Credible } \\
\text { Intervals }\end{array}$ \\
\hline \multicolumn{3}{|l|}{ Zip Code-level Percent Black (ref=lowest tertile) } \\
\hline Tertile 2 & 0.85 & $0.74-0.98$ \\
\hline Tertile 3 & 0.81 & $0.69-0.96$ \\
\hline \multicolumn{3}{|l|}{ Zip Code-level Percent White (ref=lowest tertile) } \\
\hline Tertile 2 & 0.93 & $0.81-1.08$ \\
\hline Tertile 3 & 1.13 & $0.94-1.34$ \\
\hline \multicolumn{3}{|l|}{ Zip Code-level Percent Hispanic (ref=lowest tertile) } \\
\hline Tertile 2 & 0.92 & $0.77-1.07$ \\
\hline Tertile 3 & 0.90 & $0.74-1.06$ \\
\hline \multicolumn{3}{|l|}{ Zip Code-level Percent below Federal Poverty Level } \\
\hline \multicolumn{3}{|l|}{ (FPL) (ref=lowest tertile) } \\
\hline Tertile 2 & 0.98 & $0.83-1.16$ \\
\hline Tertile 3 & 1.02 & $0.88-1.19$ \\
\hline \multicolumn{3}{|l|}{ Zip Code-level Educational attainment (ref=HS or less) } \\
\hline Some college & 1.10 & $0.83-1.46$ \\
\hline College graduate & 1.00 & $0.83-1.19$ \\
\hline \multicolumn{3}{|l|}{ Zip Code-level Composite Percent Black/Percent } \\
\hline \multicolumn{3}{|l|}{ below FPL" (ref=Group 1) } \\
\hline Group 2 & 0.88 & $0.73-1.04$ \\
\hline Group 3 & 0.87 & $0.70-1.07$ \\
\hline \multicolumn{3}{|l|}{ County-level number of hospitals per capita ${ }^{* *}$} \\
\hline \multicolumn{3}{|l|}{ (ref=lowest tertile) } \\
\hline Tertile 2 & 1.13 & $0.97-1.31$ \\
\hline Tertile 3 & 1.30 & $1.07-1.58$ \\
\hline \multicolumn{3}{|l|}{ State-level number of rheumatologists per capita* } \\
\hline \multicolumn{3}{|l|}{ (ref=lowest tertile) } \\
\hline Tertile 2 & 1.22 & $0.95-1.55$ \\
\hline Tertile 3 & 1.16 & $0.89-1.47$ \\
\hline \multicolumn{3}{|c|}{$\begin{array}{l}\text { Each variable was examined in a separate model adjusted for age, sex, race/ethnicity, SLE } \\
\text { risk adjustment index, lupus nephritis, diabetes, antidepressant use, corticosteroid use, } \\
\text { immunosuppressive use, number of lab tests, number of medications, healthcare utilization, } \\
\text { calendar year at index date, and random effects at each level. Bolded } 95 \% \text { credible inter- } \\
\text { vals do not cross } 1 \text { and Bayesian p-value was }<0.05 \text {. } \\
\text { "Group } 1=\text { zip codes with the lowest tertiles of percent black and percent below FPL, Group }\end{array}$} \\
\hline \multicolumn{3}{|c|}{$\begin{array}{l}\text { 3=zip codes with the highest tertiles of } \% \text { black and } \% \text { below FPL } \\
{ }^{* *} \text { Models also adjusted for zip code percent black }\end{array}$} \\
\hline
\end{tabular}

Methods We identified SLE patients with new use of HCQ (no use in $\geq 6$ months) in Medicaid (2000-2010) from $28 \mathrm{U}$. S. states. We required 12 months of continuous enrollment after HCQ initiation with complete drug dispensing data and measured adherence using the proportion of days covered 
(PDC) during this period. We identified individual-level variables from Medicaid, zip code, county and state-level sociodemographic variables from the American Community Survey, and health resource data from Area Health Resources Files. We used 4-level hierarchical multivariable logistic regression models to examine odds (OR (95\% Credible Interval)) of adherence (PDC $\geq 80 \%$ ) vs nonadherence.

Results Among 10,268 HCQ initiators with SLE, 15\% were adherent (PDC $\geq 80 \%$ ). After adjusting for individual-level demographic and SLE-related characteristics, we observed lower odds of adherence in zip codes with higher percentages of black individuals (highest tertile OR $0.81 \quad(0.69$ 0.96) vs lowest) (table 1). This association persisted after controlling for zip code educational attainment, percent below federal poverty level (FPL), urbanicity and healthcare resources. We did not find statistically significant associations with zip code-level percent Hispanic, percent White, education or percent below FPL. Odds of adherence were higher in counties with the highest concentration of hospitals vs the fewest (OR 1.30 (1.07-1.58)); no statistically significant associations were found with other health resource characteristics.

Conclusions Among Medicaid beneficiaries with SLE, we observed significant effects of racial composition and hospital concentration on HCQ adherence. Further studies with smaller geographic units and data on spatial relationships are needed to investigate the potential role of residential racial segregation on adherence. Interventions that acknowledge and address contextual factors should be considered to reduce high rates of nonadherence in vulnerable populations.

\section{CS-12 OUTCOMES OF LUPUS NEPHRITIS IN VULNERABLE POPULATIONS}

Christine Peschken*, Rebecca Gole, Carol A Hitchon, David Robinson, Ada Man, Annaliese Tisseverasinghe, Hani El-Gabalawy. Max Rady College of Medicine, Rady Faculty of Health Sciences, University of Manitoba, Winnipeg, Manitoba, Canada

\subsection{6/lupus-2018-Ism.47}

Background Lupus nephritis is a known predictor of mortality; we examined the risks of end-stage renal disease (ESRD) and death among lupus nephritis patients, and included the impact of ethnicity, low income (LowInc), lack of education (LowEduc), and living $>500 \mathrm{~km}$ from rheumatology care (Remote).

Methods Patients from a single academic center were followed from 1990-2016 using a custom database. Records of all SLE patients were abstracted. Variables included birthdate, diagnosis date, ethnicity, ACR classification criteria (ACRc), SLICC Damage Index (SDI) including ESRD, treatment and date of death. Ethnicity was categorized into North American Indigenous (IND), Asian (ASN), Caucasian (CAU), and Other. In patients who had developed nephritis, Kaplan Meier and Cox proportional hazard models were used to compare ESRD and survival between vulnerable groups.

Results Nine hundred forty-four SLE patients were identified: 240 (25\%) IND, 576(60\%) CAU, 104(11\%) ASN and 24 $(2.5 \%)$ Other. 'Other' patients were excluded from further analysis. Mean disease duration was 14 years, $89 \%$ female. Nephritis developed in $39 \%$ of CAU $(n=224), 57 \%$ of IND ( $n=136$; OR $2.1 ; 95 \%$ CI 1.5 to 2.8$)$, and $75 \%$ of ASN $(n=76$; OR $4.7 ; 95 \%$ CI 2.9 to 7.6$), p<0.001$. Twenty percent of patients had not completed high school, 20\% were LowInc, and $11 \%$ were Remote; LowInc, LowEduc, and Remote did not increase the odds of nephritis. Among nephritis patients, ESRD developed in $11 \%$ and $17 \%$ died. Risk of ESRD was increased in IND (HR 4.2; 95\% CI 2.0 to 8.6) and ASN (HR 5.8; 95\% CI 2.6 to 12.6) compared to CAU (figure 1a). Risk of death was increased in IND (HR 2.6; 95\% CI 1.6 to 4.2), but not in ASN (HR 1.1; 95\% CI 0.5 to 2.4) compared to CAU (figure $1 \mathrm{~b}$ ). In separate cox proportional hazards models, after adjustment for age, gender, SDI, ACRc, and age at diagnosis, risk of ESRD was increased in NAI (HR 2.8; 95\% CI 1.0 to 8.1) and ASN (HR 4.0; 95\% CI 1.6 to 10.3$)$ compared to CAU. LowInc, LowEduc, and Remote did not increase risk of ESRD. Only LowEduc (HR 2.1; 95\% CI 1.1 to 3.9) increased the adjusted risk of death; ethnicity, LowInc and Remote were not significant.

Conclusions Compared to CAU, IND and ASN not only have a higher risk of nephritis, but among those with nephritis, risk of ESRD is 3-4 fold higher in IND and ASN. Lack of education, rather than ethnicity, was the major risk factor for death. Reasons for these differences may include renal pathology, care pathways, comorbid conditions and additional socioeconomic factors and need to be further explored.

Acknowledgements We gratefully acknowledge the Lupus Society of Manitoba for ongoing funding for this work.
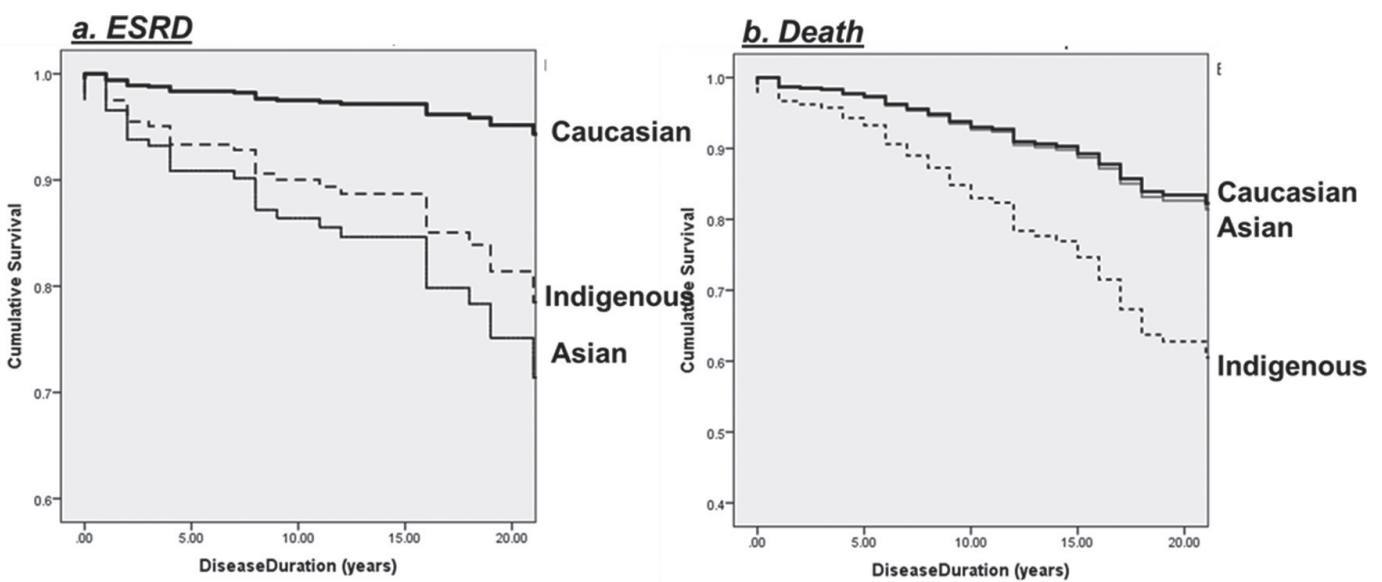

Abstract CS-12 Figure 1 Risk of end stage renal disease and death by ethnicity 